Sociologie et sociétés

\title{
La spécificité du champ scientifique et les conditions sociales du progrès de la raison
}

\section{Pierre BOURDIEU}

Volume 7, numéro 1, mai 1975

Science et structure sociale

URI : https://id.erudit.org/iderudit/001089ar

DOI : https://doi.org/10.7202/001089ar

Aller au sommaire du numéro

\section{Éditeur(s)}

Les Presses de l'Université de Montréal

ISSN

0038-030X (imprimé)

1492-1375 (numérique)

Découvrir la revue

Citer cet article

BOURDIEU, P. (1975). La spécificité du champ scientifique et les conditions sociales du progrès de la raison. Sociologie et sociétés, 7(1), 91-118.

https://doi.org/10.7202/001089ar
Résumé de l'article

En opposition à la sociologie officielle de la science qui ne fait qu'enregistrer l'image triomphante que l'hagiographie scientifique produit et propage, qui constitue la " communauté scientifique " comme un champ social d'exception et qui néglige les phénomènes de domination, l'auteur introduit et élabore la notion de " champ scientifique " qu'il définit à la fois comme le système des relations objectives entre des positions sociales et comme le lieu de la concurrence qui a pour enjeu spécifique le monopole de l'autorité scientifique inséparablement définie comme capacité technique et comme pouvoir social. Il découle de cette définition qu'il est vain de distinguer entre des déterminations proprement scientifiques et des déterminations proprement sociales des pratiques scientifiques : la lutte pour l'autorité scientifique est une lutte irréparablement politique et scientifique dont la spécificité est qu'elle oppose des producteurs qui tendent à n'avoir d'autres clients que leurs concurrents. 


\section{La spécificité du champ scientifique et les conditions sociales du progrès de la raison}

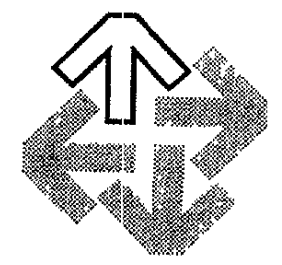

PIERRE BOURDIEU

* La formation de l'esprit scientifique est non seulement une réforme de la connaissance vulgaire, mais encore une conversion des intérêts."

Gaston BACHELARD

Le rationalisme appliqué

La sociologie de la science repose sur le postulat que la vérité du produit, s'agirait-il de ce produit très particulier qu'est la vérité scientifique —, réside dans une espèce particulière de conditions sociales de production ; c'est-à-dire, plus précisément, dans un état déterminé de la structure et du fonctionnement du champ scientifique. L'univers «pur» de la science la plus «pure » est un champ social comme un autre, avec ses rapports de forces et ses monopoles, ses luttes et ses stratégies, ses intérêts et ses profits, mais où tous ces invariants revêtent des formes spécifiques.

1. Le champ scientifique comme système des relations objectives entre les positions acquises (par les luttes antérieures) est le lieu (c'est-à-dire l'espace de jeu) d'une lutte de concurrence qui a pour enjeu spécifique le monopole de l'autorité scientifique inséparablement définie comme capacité technique et comme pouvoir 
social, ou si l'on préfère, le monopole de la compétence scientifique, entendue au sens de capacité de parler et d'agir légitimement (c'est-à-dire de manière autorisée et avec autorité) en matière de science, qui est socialement reconnue à un agent déterminé.

Deux remarques rapides, pour écarter des malentendus possibles. Premièrement, il faut se garder de réduire les relations objectives qui sont constitutîves du champ à l'ensemble des interactions, au sens de l'interactionnisme, c'est-à-dire à l'ensemble des stratégies qu'en réalité il détermine, comme on le verra plus loin (cf. P. Bourdieu, «Une interprétation de la sociologie religieuse de Max Weber », Archives européennes de sociologie, XII, 1, 1971, pp. 3-21). D'autre part, il faudra préciser ce que veut dire socialement reconnu : on verra que le groupe qui accorde cette reconnaissance tend toujours davantage à se réduire à l'ensemble des savants, c'est-à-dire des concurrents, à mesure que s'accroissent les ressources scientifiques accumulées et, corrélativement, l'autonomie du champ.

Dire que le champ est un lieu de luttes, ce n'est pas seulement rompre avec l'image irénique de la «communauté scientifique » telle que la décrit l'hagiographie scientifique - et souvent, après elle, la sociologie de la science —, c'est-à-dire avec l'idée d'une sorte de « règne des fins» qui ne connaîtrait pas d'autres lois que celle de la concurrence pure et parfaite des idées, infailliblement tranchée par la force intrinsèque de l'idée vraie. C'est aussi rappelcr que le fonctionnement même du champ scientifique produit et suppose une forme spécifique d'intérêt (les pratiques scientifiques n'apparaissant comme « désintéressées » que par référence à des intérêts différents, produits et exigés par d'autres champs).

En parlant d'intérêt scientifique et d'autorité (ou de compétence) scientifique, on entend écarter d'emblée des distinctions qui hantent, à l'état implicite, les discussions sur la science : ainsi, essayer de dissocier dans la compétence (ou l'autorité) scientifique ce qui serait pure représentation sociale, pouvoir symbolique, marqué par tout un «appareil » (au sens de Pascal) d'emblèmes et de signes, et ce qui serait pure capacité technique, c'est tomber dans le piège constitutif de toute compétence, raison sociale qui se légitime en se présentant comme pure raison technique (comme on voit par exemple dans les usages technocratiques de la notion de compétence) ${ }^{1}$. En fait, "l'appareil auguste $»$ dont sont entourés ceux que l'on appelait les « capacités » au siècle dernier et aujourd'hui les « compétences», robes rouges et hermine, soutanes et bonnets carrés des magistrats et des docteurs en un autre temps, titres scolaires et distinctions scientifiques des chercheurs aujourd'hui, toute cette «montre si authentique », comme disait Pascal, toute cette fiction sociale qui n'a rien de fictif socialement, modifie la perception sociale de la capacité proprement technique. C'est ainsi que les jugements sur les capacités scientifiques d'un

1. Le conflit que rapporte Sapolsky entre les partisans de la fluoridation, c'est-à-dire entre les détenteurs de l'autorité officielle (health officials) qui s'estiment seuls « compétents» en matière de santé publique, et les adversaires de cette innovation, parmi lesquels on compte beaucoup de scientifiques, mais qui, aux yeux des officiels, outrepassent "les limites de leur domaine propre de compétence $"$, permet de percevoir clairement la vérité sociale de la compétence, comme parole autorisée et parole d'autorité qui est l'enjeu d'une lutte entre les groupes (cf. H.M. Sapolsky, "Science, Voters and the Fluoridation Controversy", Science, vol. 162, 25 October, pp. 427-433). Le problème de la compétence ne se pose jamais avec autant d'acuité et de clarté que dans la relation avec les «profanes" (cf. S.B. Barnes, "On the Reception of Scientific Beliefs", in B. Barnes (ed.), Sociology of Science, London, Penguin, 1972, pp. 269-291; L. Boltanski et P. Maldidier, "Carrière scientifique, morale scientifique et vulgarisation ", Information sur les sciences sociales, IX, 3, 1970, pp. 99-118). 
étudiant ou d'un chercheur sont toujours contaminés, à tous les niveaux du cursus, par la connaissance de la position qu'il occupe dans les hiérarchies instituées (celle des universités par exemple aux USA) ${ }^{2}$.

Du fait que toutes les pratiques sont orientées vers l'acquisition de l'autorité scientifique (prestige, reconnaissance, célébrité, etc.), enjeu intrinsèquement double, ce que l'on appelle communément «l'intérêt »pour une activité scientifique (une discipline, un secteur de cette discipline, une méthode, etc.) est toujours à double face ; $\epsilon$ te même les stratégies qui tendent à assurer la satisfaction de cet intérêt.

Une analyse qui essayerait d'isoler une dimension purement " politique " dans les conflits pour la domination du champ scientifique serait aussi radicalement fausse que le parti-pris inverse, plus fréquent, de ne retenir que les déterminations "pures " et purement intellectuelles des conflits scientifiques. Par exemple, la lutte qui oppose aujourd'hui les spécialistes pour l'obtention de crédits et d'instruments de recherche ne se réduit jamais à une simple lutte pour le pouvoir proprement "politique ": ceux qui, dans les sciences sociales, se portent à la tête des grandes bureaucraties scientifiques ne peuvent imposer leur victoire comme une victoire de la science que s'ils se montrent capables d'imposer une définition de la science impliquant que la bonne manière de faire la science suppose l'utilisation des services d'une grande bureaucratie scientifique, pourvue de crédits, d'équipements techniques puissants, d'une main-d'œuvre abondante; et ils constituent en méthodologie universelle et éternelle les procédures de l'enquête par sondage auprès de vastes échantillons, les opérations de l'analyse statistique des données et de la formalisation des résultats instaurant ainsi en mesure de toute pratique scientifique l'étalon le plus favorable à leurs capacités personnelles et institutionnelles. Réciproquement, les conflits épistémologiques sont toujours, inséparablement, des conflits politiques : c'est ainsi qu'une enquête sur le pouvoir dans le champ scientifique pourrait parfaitement ne comporter que des questions d'allure épistémologique.

Il découle d'une définition rigoureuse du champ scientifique comme espace objectif d'un jeu où se trouvent engagés des enjeux scientifiques, qu'il est vain de distinguer entre des déterminations proprement scientifiques et des déterminations proprement sociales de pratiques essentiellement surdéterminées. Il faut citer tout un passage de la description de Fred Reif qui fait voir, presque malgré lui, combien est artificielle, voire impossible, la distinction de l'intérêt intrinsèque et de l'intérêt extrinsèque, de ce qui est important pour un chercheur déterminé et de ce qui est important pour les autres chercheurs : «A scientist strives to do research which he considers important. But intrinsic satisfaction and interest are not his only reasons. This becomes apparent when one observes what happens if the scientist discovers that someone else has just published a conclusion which he was about to reach as a result of his own research. Almost invariably he feels upset by this occurrence, although the intrinsic interest of his work has certainly not been affected. The scientist wants his work to be not only interesting himself but also important to others $\gg^{3}$. Ce qui est perçu comme important et intéressant, c'est ce qui a des

2. C'est ce que dit bien un physicien dans un article tout à fait remarquable - qui contraste par sa lucidité et sa netteté avec le gros de la littérature sociologique consacrée à la science - : "While still in high school, the scientist-to-be becomes aware that competition and prestige will affect his future success. He must strive for good grades in order to be admitted to college and later to graduate school. He realizes the importance of attending a college of high reputation not only because it will provide him with a better education but also because it will facilitate his later admission to a good graduate school. " (F. Reif, "The Competitive World of the Pure Scientist", Science, 15 déc. 1961, vol. 134, n 3494, pp. 1957-1962).

3. F. Reif, loc. cit. 
chances d'être reconnu comme important et intéressant par les autres, donc de faire apparaître celui qui le produit comme important et intéressant aux yeux des autres (il faudra examiner à nouveau cette dialectique et les conditions dans lesquelles elle fonctionne au profit de la cumulativité scientifique et non comme un simple cercle de légitimation mutuelle).

Sous peine de revenir à la philosophie idéaliste qui accorde à la science le pouvoir de se développer selon sa logique immanente (comme le fait encore Kuhn lorsqu'il suggère que les « révolutions scientifiques » ne surviennent qu'à la suite de l'épuisement des «paradigmes $\gg)$, il faut supposer que les investissements s'organisent par référence à une anticipation - consciente ou inconsciente - des chances moyennes de profit (qui se spécifient encore en fonction du capital détenu). C'est ainsi que la tendance des chercheurs à se concentrer sur les problèmes considérés comme les plus importants (par exemple parce qu'ils ont été constitués comme tels par des producteurs dotés d'un haut degré de légitimité) s'explique par le fait qu'un apport ou une découverte concernant ces questions est de nature à apporter un profit symbolique plus important. La compétition intense qui se trouve ainsi déclenchée a toutes chances de déterminer une baisse des taux moyens de profit symbolique et par là le départ d'une fraction des chercheurs vers d'autres objets moins prestigieux mais autour desquels la compétition est moins forte, donc propres à offrir des profits au moins aussi importants ${ }^{4}$.

La distinction que fait Merton entre des conflits "sociaux " (portant sur "l'allocation des ressources intellectuelles entre différentes sortes de travail sociologique " ou sur " le rôle qui convient au sociologue ") et des conflits " intellectuels ", " oppositions d'idées sociologiques strictement formulées * (R. K. Merton, The Sociology of Science, Chicago and London, The University of Chicago Press, 1973, p. 55), constitue elle-même une stratégie à la fois sociale et intellectuelle qui tend à imposer une délimitation du champ des objets de discussion légitimes. On aura en effet reconnu dans cette distinction une de ces stratégies par lesquelles la sociologie américaine officielle tend à s’assurer la respectabilité académique et à imposer une délimitation du scientifique et du non-scientifique propre à interdire comme manquement à la bienséance scientifique toute interrogation propre à mettre en question les fondements de sa respectabilité 5 .

Une science authentique de la science ne peut se constituer qu'à condition de récuser radicalement l'opposition abstraite (qui se retrouve aussi ailleurs, en histoire de l'art par exemple) entre une analyse immanente ou interne, qui incomberait en propre à l'épistémologie et qui restituerait la logique selon laquelle la science engendre ses propres problèmes, et une analyse externe, qui rapporte ces problèmes à leurs conditions sociales d'apparition. C'est le champ scientifique qui, en tant que

4. C'est dans la même logique qu'il faut comprendre les transferts de capital d'un champ déterminé dans un champ inférieur, où une compétition moins intense promet des chances de profit plus élevé au détenteur d'un capital scientifique déterminé.

5. Soit parmi les innombrables expressions de ce credo neutraliste, celle-ci, particulièrement typique: "One essential notion of sociologists as professionals - either academically based or practice oriented - is that they are able to separate, in a socially responsible fashion, their personal ideology from their professional roles in dealing with their clients, their publics, and their peers. Clearly, this dimension represents the deepest and most profound issue in the application of the concept of professionalisation to sociology and especially in the period of university activism since 1965 (Ben-David 1972). Many sociologists, since the initial organization of sociology as a discipline, have had strong personal ideologies which have pressed them to seek to make their knowledge relevant or effective for social change; yet as academics they have had to face or have been attracted to the norms of the teacherresearcher» (M. Janowitz, The American Journal of Sociology, vol. 78, $\mathrm{n}^{\circ}$ 1, July 1972 , pp. 105-135). 
lieu d'une lutte politique pour la domination scientifique, assigne à chaque chercheur, en fonction de la position qu'il y occupe, ses problèmes indissociablement politiques et scientifiques et ses méthodes stratégiques scientifiques qui, du fait qu'elles se définissent expressément ou objectivement par référence au système des positions politiques et scientifiques constitutives du champ scientifique, sont en même temps des stratégies politiques. Il n'est pas de «choix » scientifique choix du domaine de recherche, choix des méthodes employées, choix du lieu de publication, choix, que décrit Hagstrom ${ }^{6}$, de la publication rapide de résultats partiellement vérifiés ou de la publication tardive de résultats pleinement contrôlés qui ne soit pas un de ses aspects, - le moins avoué et le moins avouable évidemıment -, une stratégie politique de placement au moins objectivement orientée vers la maximisation du profit proprement scientifique, c'est-à-dire de la reconnaissance susceptible d'être obtenue des pairs-concurrents.

2. La lutte pour l'autorité scientifique, espèce particulière de capital social qui assure un pouvoir sur les mécanismes constitutifs du champ et qui peut être reconverti en d'autres espèces de capital, doit l'essentiel de ses caractéristiques au fait que les producteurs tendent à n'avoir d'autres clients possibles que leurs concurrents (cela d'autant plus que le champ est plus autonome). Cela signifie que dans un champ scientifique fortement autonome, un producteur particulier ne peut attendre la reconnaissance de la valeur de ses produits ( réputation », "prestige », « autorité », « compétence », etc.) que des autres producteurs qui, étant aussi ses concus:rents, sont les moins enclins à la lui accorder sans discussion ni examen. D'abord en fait: seuls des savants engagés dans le même jeu ont les moyens de s'approprier symboliquement l'œuvre scientifique et d'en évaluer les mérites. Et aussi en droit: celui qui fait appel à une autorité extérieure au champ ne peut s'attirer que le discrédit ${ }^{\top}$. (En cela, le champ scientifique est tout à fait semblable à un champ artistique fortement autonome ${ }^{8}$ : un des principes de la spécificité du champ scientifique résidant dans le fait que les concurrents ne peuvent pas se contenter de se distinguer de leurs devanciers déjà reconnus, mais sont contraints sous peine d'être dépassés et "déclassés », d'intégrer leurs acquis dans la construction distincte et distinctive qui les dépasse).

6. W.D. Hagstrom, The Scientific Community, New York, Basic Books, 1965, p. 100.

7. Fred Reif rappelle que ceux qui, par souci de voir leur travail publié le plus rapidement possible, recourent à la presse quotidienne (des découvertes importantes en physique ont ainsi pu être annoncées dans le New York Times) s'attirent la réprobation de leurs pairsconcurrents, au nom de la distinction entre publication et publicité qui commande aussi les attitudes à l'égard de certaines formes de vulgarisation, perçues comme auto-divulgation. Il suffira de citer les commentaires de l'éditeur du journal officiel des physiciens américains : * As a matter of courtesy to fellow physicists, it is customary for authors to see to it that releases to the public do not occur before the article appears in the scientific journal. Scientific discoveries are not the proper subject for newspaper scoops, and all media of mass communication should have equal opportunity for simultaneous access to the information. In the future, we may reject papers whose main content has been published previously in the daily press (F. Reif, loc. cit.).

8. Sur ce point, voir P. Bourdieu, "Le marché des biens symboliques ", L'année sociologique, vol. 22, 1971, pp. 49-126 (les autoréférences, très nombreuses dans ce texte, ont une fonction strictement sténographique). 
La lutte dans laquelle chacun des agents doit s'engager pour imposer la valeur de ses produits et sa propre autorité de producteur légitime a toujours en fait pour enjeu le pouvoir d'imposer la définition de la science (i.e. la délimitation du champ des problèmes, des méthodes et des théories qui peuvent être considérés comme scientifiques) la plus conforme à ses intérêts spécifiques, c'est-à-dire la mieux faite pour lui permettre d'occuper en toute légitimité la position dominante en assurant la position la plus haute dans la hiérarchie des valeurs scientifiques aux capacités scientifiques dont il est le détenteur à titre personnel ou institutionnel (par exemple en tant que directeur d'une forte culture mathématique, en tant qu'ancien élève d'une institution d'enseignement particulière, en tant que membre d'une institution scientifique déterminée, etc.) ${ }^{9}$.

C'est ainsi que les débats sur la priorité des découvertes opposent en plus d'un cas celui qui a découvert le phénomène inconnu sous la forme, souvent, d'une simple anomalie, d'un raté des théories existantes, et celui qui en a fait un fait scientifique nouveau en l'insérant dans une construction théorique qui est irréductible au simple donné brut : ces discussions politiques sur le droit de propriété scientifique qui sont en même temps des débats scientifiques sur le sens de ce qui est découvert et des discussions épistémologiques sur la nature de la découverte scientifique, opposent en réalité, à travers ces protagonistes particuliers, deux principes de hiérarchisation des pratiques scientifiques, l'un qui confère la primauté à l'observation et à l'expérimentation, donc aux đispositions et aux capacités correspondantes, l'autre qui privilégie la théorie et les «intérêts " scientifiques corrélatifs, débat qui n'a jamais cessé d'occuper le centre de la réflexion épistémologique.

Ainsi, la définition de l'enjeu de la lutte scientifique fait partie des enjeux de la lutte scientifique et les dominants sont ceux qui parviennent à imposer la définition de la science selon laquelle la réalisation la plus accomplie de la science consiste à avoir, être et faire, ce qu'ils ont, sont ou font. C'est dire en passant que la communis doctorum opinio, comme disait la scolastique, n'est jamais qu'une fiction officielle qui n'a rien de fictif parce que l'efficacité symbolique que lui confère sa légitimité lui permet de remplir une fonction semblable à celle que l'idéologie libérale fait jouer à la notion d'opinion publique. La science officielle n'est pas ce qu'en fait le plus souvent la sociologie de la science, c'est-à-dire le système des normes et des valeurs que la «communauté scientifique», groupe indifférencié, imposerait et inculquerait à tous ses membres, l'anomie révolutionnaire ne pouvant s'imputer qu'à des ratés de la socialisation scientifique ${ }^{10}$. Cette vision « durkheimienne » du champ scientifique pourrait n'être que la transfiguration de la représentation naïvement «fonctionnaliste $»$ de l'univers scientifique que les tenants de l'ordre scientifique ont intérêt à imposer, et d'abord à leurs concurrents.

9. Il y a, à chaque moment, une hiérarchie sociale des champs scientifiques - les disciplines - qui oriente fortement les pratiques et tout particulièrement les "choix 》 de "vocation" - et à l'intérieur de chacun d'eux, une hiérarchie sociale des objets et des méthodes de traitement. (Sur ce point, cf. P. Bourdieu, "Méthode scientifique et hiérarchie sociale des objets ", Actes de la recherche en sciences sociales, 1, 1975, pp. 4-6).

10. Comme la philosophie sociale d'inspiration durkheimienne qui décrit le conflit social dans le langage de la marginalité, de la déviance ou de l'anomie, cette philosophie de la science tend à réduire les relations de compétition entre dominants et dominés aux relations entre un "centre" et une "périphérie", retrouvant la métaphore émanatiste chère à Halbwachs de la distance au "foyer » des valeurs centrales (cf. par exemple, J. Ben David, The Scientist's Role in Society, Englewood Cliffs (N.J.), Prentice Hall Inc., 1971, et E. Shils, "Center and Periphery", in The Logic of Personal Knowledge, Essays Presented to Michael Polanyi on His Seventieth Birthday, London, Routledge and Kegan Paul Ltd, 1961, pp. 117130 ). 
On n'en finirait pas de recenser les exemples de ce "fonctionnalisme " même chez un auteur qui, comme Kuhn, fait place au conflit dans sa théorie de l'évolution scientifique : "A cornmunity of scientific specialists will do all that it can to ensure the continuing growth of the assembled data that it can treat with precision and detail " (T. Kuhn, The Structure of Scientific Revolutions, Chicago, The University of Chicago Press, 1962, p. 168). Parce que la "fonction" au sens du "fonctionnalisme» de l'école américaine n'est autre chose que l'intérêt des dominants, c'est-à-dire l'intérêt que les dominants ont à la perpétuation d'un système conforme à leurs intérêts (ou la fonction que le système remplit pour cette classe particulière d'agents), il suffit de passer sous silence les intérêts (i.e. les fonctions différentielles) pour tomber dans le * fonctionnalisme ".

Et c'est bien parce que la définition de l'enjeu de la lutte est un enjeu de lutte, même dans des sciences - comme les mathématiques - où le consensus apparent sur les enjeux est très élevé, que l'on se heurte sans cesse aux antinomies de la légitimité. (L'intérêt passionné que les chercheurs en sciences sociales portent aux sciences de la nature ne se comprendrait pas autrement: c'est la définition des principes d'évaluation de leur propre pratique qui est en jeu dans leur prétention d'imposer, au nom de l'épistémologie ou de la sociologie de la science, la définition légitime de la forme la plus légitime de la science, c'est-à-dire la science de la nature). Dans le champ scientifique comme dans le champ des rapports de classes, il n'existe pas d'instance à légitimer les instances de légitimité ; les revendications de légitimité tiennent leur légitimité de la force relative des groupes dont elles expriment les intérêts : dans la mesure où la définition même des critères de jugement et des principes de hiérarchisation est l'enjeu d'une lutte, personne n'est bon juge parce qu'il n'est pas de juge qui ne soit juge et partie.

Cn voit la naïveté de la technique des "juges» à laquelle la tradition sociologique a très communément recours pour définir les hiérarchies caractéristiques d'un champ déterminé (hiérarchie des agents ou des institutions - les universités aux U.S.A. - , hiérarchie des problèmes, des domaines ou des méthodes, hiérarchie des champs eux-mêmes, etc.). C'est la même philosophie naïve de l'objectivité qui inspire le recours à des "experts internationaux" (cf. les rapports réalisés à la demande de l'OCDE, dont le plus récent est le rapport Hoffman, Léontiev Tajfel sur les sciences sociales en France). Comme si leur position d'observateurs étrangers pouvait les mettre à l'abri des partis pris et des prises de parti à un moment où l'économie des échanges idéologiques connaît tant de sociétés multinationales et comme si leurs analyses "scientifiques" de l'état de la science pouvait être autre chose que la justification scientifiquement masquée de l'état particulier de la science ou des institutions scientifiques avec lequel ils ont partie liée (dans l'exemple cité, l'université américaine et sa sociologie). On verra plus loin que la sociologie de la science échappe très rarement à cette stratégie de l'expertise engagée comme imposition de légitimité préparant une conquête de marché ${ }^{11}$.

L'autorité scientifique est donc une espèce particulière de capital qui peut être accumulé, transmis et même reconverti en d'autres espèces sous certaines conditions.

On peut emprunter à Fred Reif la description du processus d'accumulation du capital scientifique et des formes que prend sa reconversion. Cela dans le cas

11. Derrière les problématiques d'experts sur la valeur relative des régimes universitaires se cache inévitablement la question des conditions optimales pour le développement de la science et par là du meilleur régime politique, les sociologues américains ayant tendance à faire de la "démocratie libérale» à la manière américaine la condition de la " démocratie scientifique ». Cf. par exemple R.K. Merton, "Science and Technology in a Democratic Order ", Journal of Legal and Political Sociology, vol. 1, 1942, republié in R.K. Merton, Social Theory and Social Structure, ed. révisée, Free Press, 1967, pp. 550-551, sous le titre "Science and Democratic Social Structure", B. Barber, Science and the Social Order, Glencoe, The Free Press, 1952, pp. 73 et 83. 
particulier du champ de la physique contemporaine, où la possession d'un capital scientifique tend à favoriser l'acquisition de capital supplémentaire et où la carrière scientifique « réussie » se présente de ce fait comme un processus continu d'accumulation dans lequel le capital initial, représenté par le titre scolaire, joue un rôle déterminant: "While still in high school, the scientist-to-be-becomes aware that competition and prestige will affect his future success. He must strive for good grades in order to be admitted to college and later to graduate school. He realizes the importance of attending a college of high reputation (...) Finally he must earn the good opinion of his teachers to secure the letters of recommendation which will help him enter college and gain scholarship grants and prizes (...) The job-seeking scientist is clearly in a more advantageous situation if he comes from a well-known institution and has been associated with a scientist of reputation. Invariably it is essential to him that there should be prominent scientists in the world who are willing to comment favorably upon the quality of his work. (...) Promotion to higher academic rank is subject to simular criteria. Again the university requests letters of recommendation from outside scientists and in some cases may appoint reviewing committees before deciding to promote someone to a tenure position $\gg$. Ce processus se poursuit avec l'accès aux postes administratifs, aux commissions gouvernementales, etc. Et le chercheur dépend aussi de sa réputation auprès de ses collègues pour obtenir des fonds de recherche, pour attirer des étudiants de qualité, pour s'assurer des grants et des bourses, des invitations et des consultations, des distinctions (Prix Nobel, National Academy of Science).

La reconnaissance socialement marquée et garantie (par tout un ensemble de signes spécifiques de consécration ${ }^{12}$ que le groupe des pairs-concurrents accorde à chacun de ses membres est fonction de la valeur distinctive de ses produits et de l'originalité collectivement reconnue (au sens de la théorie de l'information) de la contribution qu'il apporte aux ressources scientifiques déjà accumulées. Le fait que le capital d'autorité procuré par la découverte soit monopolisé par le premier à l'avoir faite ou, du moins, à l'avoir fait connaître et reconnaître explique l'importance et la fréquence des questions de priorité. S'il arrive que plusieurs noms soient attachés à la première découverte, le prestige attribué à chacun d'eux s'en trouve diminué d'autant. Celui qui parvient à la découverte quelques semaines ou quelques mois après un autre a dépensé ses efforts en pure perte, ses travaux se trouvant ainsi réduits au statut de duplication sans intérêt d'un travail déjà reconnu (ce qui explique la précipitation que mettent certains à publier pour éviter d'être

12. Soit, d'après Glaser, « epinomy, prizes, awards, fellowships, scholarships, honorary memberships and committee in scientific organization, editorship, honorary degrees, professorships, chairs, lectureships, consultantships, mentions by historians of science, publication, acknowledgements in other works, evaluation by colleagues * (B. G. Glaser, Organizational Scientists: Their Professional Careers, Indianapolis, Bobbs Merrill, 1964, p. 2). Selon le même auteur, la « reconnaissance moyenne » se marque par « supervisor's favorable evaluation of the quality of the scientist's current research, and proper credit, through publication and through acknowledgement in publications of others for his contribution to the cumulative knowledge in his field". Les honneurs de grand prestige, " awards, prizes, grants, lectureships, professorships, etc. ", sont les signes de reconnaissance réservés aux "great men " (B. G. Glaser, "Comparative Failure in Science ", Science, mars 6, 1964, vol. 143, $\mathrm{n}^{\circ} 3610$, pp. 1012-1014). 
devancés) ${ }^{13}$. Le concept de visibility qu'emploient souvent les auteurs américains (il s'agit, comme souvent, d'une notion d'usage courant parmi les universitaires) exprime bien la valeur différentielle, distinctive, de cette espèce particulière de capital social : accumuler du capital, c'est «se faire un nom », un nom propre (et, pour certains, un prénom), un nom connu et reconnu, marque qui distingue d'emblée son porteur, l'arrachant comme forme visible au fond indifférencié, inaperçu, obscur, dans lequel se perd le commun (de là sans doute l'importance des métaphores perceptives, dont l'opposition entre brillant et obscur est le paradigme, dans la plupart des taxinomies scolaires) ${ }^{14}$. La logique de la distinction fonctionne à plein dans le cas des signatures multiples qui, en tant que telles, réduisent la valeur distinctive impartie à chacun des signataires. On peut ainsi comprendre l'enserable des observations de Harriet A. Zuckerman ${ }^{15}$ sur les «modèles de rang de nomination chez les auteurs d'articles scientifiques» comme le produit de stratégies visant à minimiser la perte de valeur distinctive qui est imposée par les nécessités de la nouvelle division du travail scientifique. Ainsi, pour expliquer que les lauréats de prix Nobel ne soient pas nommés plus souvent que les autres au premier rang, comme on devrait s'y attendre étant donné que les auteurs sont normalement nommés dans l'ordre de la valeur relative de leur contribution, il n'est pas besoin d'invoquer une morale aristocratique du «noblesse oblige »; il suffit en effet de supposer que la visibilité d'un nom dans une série est fonction premièrement de sa visibilité relative, définie par le rang qu'il occupe dans la série et, deuxièmement, de sa visibilité intrinsèque, qui résulte du fait que, déjà connu, il est plus aisément reconnu et retenu (un des mécanismes qui font que, ici aussi, le capittal va au capital) pour comprendre que la tendance à abandonner à d'autres le premier rang croisse à mesure que croît le capital possédé, donc le profit symboli-

13. Ainsi s'expliquent les stratégies très différentes que les chercheurs mettent en oeuvre dans la diffusion des preprints et des reprints. Il serait facile de montrer que toutes les différences observées selon la discipline et l'âge des chercheurs ou l'institution à laquelle ils appartiennent peuvent être comprises à partir des fonctions très différentes que remplissent ces deux formes de communication scientifique, la première consistant à diffuser très rapidement en échappant aux délais de la publication scientifique (avantage important dans les secteur's hautement compétitifs), auprès d'un nombre restreint de lecteurs qui sont souvent aussi les concurrents les plus compétents, des produits qui ne sont pas protégés par la publication officielle contre l'appropriation frauduleuse mais qui sont susceptibles d'être améliorés par la circulation, la seconde consistant à divulguer plus largement, auprès de l'ensemble des collègues ou des demandeurs, des produits marqués et socialement imputés à un nom propre (cf. W. Hagstom, "Factors Related to the Use of Different Modes of Publishing Research in Four Scientific Fields 》, in C.E. Nelson et D.K. Pollock, eds., Communication Among Scientists and Engineers, Lexington (Mass.), Health Lemington Books, D.C. Heath and $\mathrm{Co}, 1970$.

14. De là les difficultés que l'on rencontre dans les recherches sur les intellectuels, les savants ou les artistes, tant dans l'enquête elle-même que dans la publication des résultats : proposer l'anonymat à des gens qui sont tout occupés à se faire un nom, c'est faire disparaître la motivation principale de la participation à une enquête (cf. le modèle de l'enquête littéraire ou de l'interview); ne pas le proposer c'est s'interdire de poser des questions " indiscrètes ", c'est-à-dire objectivantes et réductrices. La publication des résultats pose des problèmes équivalents, ne serait-ce que parce que l'anonymat a pour effet de rendre le discours inintelligible ou transparent selon le degré d'information des lecteurs (cela d'autarit plus que, dans ce cas, certains positions peuvent ne compter qu'un élément, un nom propre).

15. H.A. Zuckerman, "Patterns of Name Ordering among Authors of Scientific Papers : A study of Social Symbolism and its Ambiguity », American Journal of Sociology, vol. 74, $n^{\circ} 3$, nov. 1968, pp. 276-291. 
que automatiquement assuré à son possesseur, indépendamment du rang de nomination ${ }^{16}$. Le marché des biens scientifiques a ses lois, qui n'ont rien à voir avec la morale. Et sous peine de faire entrer dans la science de la science, sous divers noms «savants», ce que les agents appellent parfois « les valeurs» ou «les traditions » de «la communauté scientifique », il faut savoir reconnaître pour telles les stratégies qui, dans les univers où l'on a intérêt au désintéressement tendent à dissimuler les stratégies. Ces stratégies du second ordre, par lesquelles on se met en règle en transfigurant la soumission aux lois (qui est la condition de la satisfaction des intérêts) en obéissance élective aux normes, permettent de cumuler les satisfactions de l'intérêt bien compris et les profits à peu près universellement promis à des actions sans autre détermination apparente que le respect pur et désintéressé de la règle.

3. La structure du champ scientifique est définie à chaque moment par l'état $\mathrm{du}$ rapport de forces entre les protagonistes de la lutte, agents ou institutions, c'est-à-dire par la structure de la distribution du capital spécifique, résultat des luttes antérieures qui se trouve objectivé dans des institutions et des dispositions et qui commande les stratégies et les chances objectives des différents agents ou institutions dans les luttes présentes. (Il suffit, ici comme ailleurs, d'apercevoir la relation dialectique qui s'établit entre les structures et les stratégies - par l'intermédiaire des dispositions - pour faire disparaître l'antinomie de la synchronie et de la diachronie, de la structure et de l'histoire dans laquelle s'enferment l'objectivisme structuraliste et le subjectivisme spontanéiste). La structure de la distribution du capital scientifique est au principe des transformations du champ scientifique par l'intermédiaire des stratégies de conservation ou de subversion de la structure que la structure elle-même produit: d'une part la position que chaque agent singulier occupe à un moment donné du temps dans la structure du champ scientifique est la résultante, «cristallisée » dans des institutions et des dispositions, de l'ensemble des stratégies antérieures de cet agent et de ses concurrents qui dépendent elles-mêmes de la structure du champ par l'intermédiaire des propriétés structurales de la position à partir de laquelle elles sont engendrées; et d'autre part les transformations de la structure du champ sont le produit de stratégies de conservation ou de subversion qui trouvent le principe de leur orientation et de leur efficacité dans les propriétés de la position qu'occupent ceux qui les produisent à l'intérieur de la structure du champ.

Cela signifie que dans un état déterminé du champ, les investissements des chercheurs dépendent tant dans leur importance (mesurable par exemple en temps consacré à la recherche) que dans leur nature (et en particulier, dans le degré de risque assumé) de l'importance de leur capital actuel et potentiel de reconnaissance et de leur position actuelle et potentielle dans le champ (selon un processus circulaire qui s'observe dans tous les domaines de la pratique). Selon une logique

16. Le modèle proposé ici rend compte parfaitement - sans faire appel à aucun déterminant moral - du fait que les lauréats cèdent le premier rang plus souvent après l'obtention du prix et que leur contribution à la recherche couronnée soit plus visiblement marquée que la part qu'ils ont prise à leurs autres recherches collectives. 
mainte fois observée, les aspirations - c'est-à-dire ce que l'on appelle communément les « ambitions scientifiques» - sont d'autant plus hautes que le capital de reconnaissance est plus élevé : la possession du capital que confère dès l'origine de la carrière scientifique le système scolaire sous la forme d'un titre rare implique et impose - par des médiations complexes - la poursuite d'objectifs élevés qui sont socialement appelés et garantis par ce titre. Ainsi, essayer de mesurer la relation statistique qui s'établit entre le prestige d'un chercheur et le prestige de ses titres scolaires d'origine (grande école ou faculté en France, université où a été décerné le doctorat pour les USA) une fois contrôlés les effets de sa productivité ${ }^{17}$, c'est assumer implicitement l'hypothèse que la productivité et le prestige actuel sont indépendants (entre eux) et inđépendants des titres d'origine: en fait, dans la mesure où le titre, en tant que capital scolaire reconvertible en capital universitaire et scientifique enferme une trajectoire probable, il commande, par l'intermédiaire des « aspirations raisonnables » qu'il autorise, tout le rapport à la carrière scientifique (le choix d'objets plus ou moins «ambitieux», une productivité plus ou moins grande, etc.) ; en sorte que l'effet du prestige des institutions ne s'exerce pas seulement de manière directe, en «contaminant» le jugement porté sur les capacités scientifiques manifestées par la quantité et la qualité des travaux, ou même de manière indirecte, par l'intermédiaire des contacts avec les maîtres les plus prestigieux que procure une haute origine scolaire (le plus souvent associée à une haute origine sociale), mais aussi par l'intermédiaire de la «causalité du probable », c'est-à-dire par la vertu des aspirations qu'autorisent ou favorisent les chances objectives (on pourrait faire des observations analogues à propos des effets de l'origine sociale à titre scolaire de départ semblable). C'est ainsi par exemple que l'opposition entre les placements sûrs de la recherche intensive et spécialisée et les placements risqués de la recherche extensive qui peut conduire aux vastes synthèses théoriques (révolutionnaires ou éclectiques) - ceux qui, dans le cas de la physique analysé par Fred Reif, consistent à s'informer sur les développements scientifiques survenus hors des limites strictes de la spécialité au lieu de rester sur les sentiers battus d'une direction de recherche éprouvée, et qui peuvent rester en pure perte ou fournir des analogies fécondes - tend à reproduire l'opposition entre les trajectoires hautes et les trajectoires basses dans le champ scolaire et dans le champ scientifique ${ }^{18}$. De même, pour comprendre la transformation souvent décrite des pratiques scientifiques qui accompagne l'avancement dans la carrière scientifique, il faut rapporter les différentes stratégies scientifiques - par exemple les investissements massifs et extensifs dans la seule recherche ou les investissements modérés et intensifs dans la recherche associés à des investissements dans l'administration scientifique - non pas évidemment à des classes d'âge chaque champ définissant ses lois propres de vieillissement social ${ }^{10}$ - mais à

17. Cf. par exemple L. L. Hargens et W. O. Hagstrom, "Sponsored and Contest Mobility of Anerican Academic Scientists ", Sociology of Education, vol. 40, $\mathrm{n}^{\circ}$, Winter 1967, pp. 24-38.

18. Cf. P. Bourdieu, L. Boltanski et P. Maldidier, "La défense du corps ", Information sur les sciences sociales, $10, \mathrm{n}^{\circ} 4$, pp. 45-86.

19. L'analyse statistique montre par exemple que, pour l'ensemble des générations passées, lâge de productivité scientifique maximum se situait entre 26 et 30 chez les chimistes, entre 30 et $34 \mathrm{chez}$ les physiciens et les mathématiciens, entre 35 et 39 chez les bactériologistes, les géologues et les physiologistes (H.C. Lehman, Age and Achievment, Princeton, Princeton University Press, 1953). 
l'importance du capital scientifique possédé qui, en définissant à chaque moment les chances objectives de profit, définit les stratégies « raisonnables» d'investissement et de désinvestissement. Rien n'est plus artificiel, on le voit, que de décrire les propriétés génériques des différentes phases de la «carrière scientifique ${ }^{20}$, s'agirait-il de la "carrière moyenne » dans un champ particulier ${ }^{21}$ - parce que toute carrière se définit fondamentalement par la position qu'elle occupe dans la structure du système des carrières possibles ${ }^{22}$. Il existe autant de manières d'entrer dans la recherche, de se maintenir dans la recherche et de sortir de la recherche qu'il y a de classes de trajectoires et toute description qui, s'agissant d'un tel univers, s'en tient aux caractéristiques génériques d'une carrière «quelconque » fait disparaître l'essentiel, c'est-à-dire les différences. La décroissance avec l'âge de la quantité et de la qualité des productions scientifiques que l'on observe dans le cas des «carrières moyennes » et qui se comprend apparemment si l'on admet que l'accroissement du capital de consécration tend à réduire l'urgence de la haute productivité qui a été nécessaire pour l'obtenir, ne devient intelligible complètement que si on réfère les carrières moyennes aux carrières les plus hautes, qui sont seules à procurer jusqu'au bout les profits symboliques nécessaires pour réactiver continûment la propension à de nouveaux investissements, retardant ainsi continûment le désinvestissement.

4. La forme que revêt la lutte inséparablement politique et scientifique pour la légitimité scientifique dépend de la structure du champ, c'est-à-dire de la structure de la distribution du capital spécifique de reconnaissance scientifique entre les participants à la lutte. Cette structure peut théoriquement varier (comme c'est aussi le cas en tout champ) entre deux limites théoriques, en fait jamais atteintes, soit d'un côté la situation de monopole du capital spécifique d'autorité scientifique et, de l'autre, la situation de concurrence parfaite supposant la distribution égale de ce capital entre tous les concurrents. Le champ scientifique est toujours le lieu d'une lutte, plus ou moins inégale, entre des agents inégalement pourvus de capital spécifique, donc inégalement en mesure de s'approprier le produit du travail scientifique (et aussi, en certains cas, les profits externes tels que les gratifications économiques ou proprement politiques) que produisent, par leur collaboration objective, l'ensemble des concurrents en mettant en cuvre l'ensemble des moyens de production scientifique disponibles. Dans tout champ s'opposent, avec des forces plus ou moins inégales selon la structure de la distribution du capital dans le champ (degré d'homogénéité), les dominants, occupant les positions les plus hautes dans la structure de la distribution du capital scientifique, et les dominés,

20. Cf. F. Reif and A. Strauss, "The Impact of Rapid Discovery upon the Scientist's Career ", Social Problems, vol. 12, $\mathrm{n}^{\circ}$ 3, Winter 1965, pp. 297-311.

21. Cf. B. G. Glazer, "Variations in the Importance of Recognition in Scientist's Careers ", Social Problems, Winter 1963, vol. 10, n 3, pp. 268-276.

22. Pour éviter de refaire ici toute la démonstration, je me contenterai de renvoyer à P. Bourdieu, "Les catégories de l'entendement professoral ", Actes de la recherche en sciences sociales, $\mathrm{n}^{\circ} 3,1975$, pp. 68-93. 
c'est-à-dire les nouveaux entrants, qui possèdent un capital scientifique d'autant plus important (en valeur absolue) que les ressources scientifiques accumulées dans le champ sont plus importantes.

Tout semble indiquer que, à mesure que les ressources scientifiques accumulées s'accroissent et que, en conséquence de l'élévation corrélative du droit d'entrée, s'élève le degré d'homogénéité entre les concurrents (qui, sous l'effet de facteurs indépendants, tendent à devenir de plus en plus nombreux), la compétition scientifique tend à revêtir une forme très différente dans sa forme et son intensité de celle qui s'observe dans des états plus anciens des mêmes champs ou en d'autres champs où les ressources accumulées sont moins importantes et le degré d'hétérogénéité plus grand. Ce sont ces propriétés structurales et morphologiques des différents champs que les sociologues de la science oublient le plus souvent de prendre en compte s'exposant ainsi à universaliser le cas particulier. C'est ainsi que l'opposition entre les stratégies de conservation et les stratégies de subversion qui sera analysée ci-dessous tend à s'affaiblir à mesure que l'homogénéité du champ s'accroît et que décroît corrélativement la probabilité des grandes révolutions périodiques au profit des innombrables petites révolutions permanentes.

Dans la lutte qui les oppose, les dominants et les prétendants, c'est-à-dire les nouveaux entrants, comme disent les économistes, recourent à des stratégies antagonistes, profondément opposées dans leur logique et dans leur principe : les intérêts (au double sens) qui les animent et les moyens qu'ils peuvent mettre en œuvre pour les satisfaire dépendent en effet très étroitement de leur position dans le champ, c'est-à-dire de leur capital scientifique et du pouvoir qu'il leur donne sur le champ de production et de circulation scientifique et sur les profits qu'il produit. Les dominants sont voués à des stratégies de conservation visant à assurer la perpétuation de l'ordre scientifique établi avec lequel ils ont partie liée. Cet ordre ne se réduit pas, comme on le croit communément, à la science officielle, ensemble de ressources scientifiques héritées du passé qui existent à l'état objectivé, sous forme d'instruments, d'ouvrages, d'institutions, etc. et à l'état incorporé, sous forme d'habitus scientifiques, systèmes de schèmes générateurs de perception, d'appréciation et d'action qui sont le produit d'une forme spécifique d'action pédagogique et qui. rendent possible le choix des objets, la solution des problèmes et l'évaluation des solutions. Il englobe aussi l'ensemble des institutions chargées d'assurer la production et la circulation des biens scientifiques en même temps que la repro-duction et la circulation des producteurs (ou des reproducteurs) et des consommateurs de ces biens, c'est-à-dire au premier chef le système d'enseignement, seul capable d'assurer à la science officielle la permanence et la consécration en l'inculquant systématiquement (habitus scientifiques) à l'ensemble des destinataires légitimes de l'action pédagogique et, en particulier, à tous les nouveaux entrants dans le champ de production proprement dit. Outre les instances spécifiquement chargées de la consécration (académies, prix, etc.), il comprend aussi les instruments de diffusion, et en particulier les revues scientifiques qui, par la sélection qu'elles opèrent en fonction des critères dominants, consacrent les productions conformes aux principes de la science officielle, offrant ainsi continûment l'exemple 
de ce qui mérite le nom de science, et exercent une censure de fait sur les productions hérétiques soit en les rejetant expressément, soit en décourageant purement l'intention de publication par la définition du publiable qu'elles proposent ${ }^{23}$.

C'est le champ qui assigne à chaque agent ses stratégies, s'agirait-il de celle qui consiste à renverser l'ordre scientifique établi. Selon la position qu'ils occupent dans la structure du champ (et sans doute aussi selon des variables secondaires telles que la trajectoire sociale, qui commande l'évaluation des chances), les «nouveaux entrants $\gg$ peuvent se trouver orientés vers les placements sûrs des stratégies de succession, propres à leur assurer, au terme d'une carrière prévisible, les profits promis à ceux qui réalisent l'idéal officiel de l'excellence scientifique au prix d'innovations circonscrites dans les limites autorisées, ou vers des stratégies de subversion, placements infiniment plus coûteux et plus risqués qui ne peuvent assurer les profits promis aux détenteurs du monopole de la légitimité scientifique qu'au prix d'une redéfinition complète des principes de légitimation de la domination : les nouveaux entrants qui refusent les carrières tracées ne peuvent «battre les dominants à leur propre jeu » qu'à condition d'engager un surcroît d'investissements proprement scientifiques et sans pouvoir en attendre des profits importants, au moins à court terme, puisqu'ils ont contre eux toute la logique du système.

D'un côté, l'invention selon un art d'inventer déjà inventé qui, en résolvant tous les problèmes susceptibles d'être posés dans les limites de la problématique établie par l'application de méthodes attestées (ou en travaillant à sauver les principes contre les contestations hérétiques - on pense par exemple à Tycho Brahé -) tend à faire oublier qu'elle ne résoud que les problèmes qu'elle peut poser ou qu'elle ne pose que les problèmes qu'elle peut résoudre; de l'autre, l'invention hérétique qui, en mettant en question les principes mêmes de l'ordre scientifique ancien, instaure une alternative tranchée, sans compromis possible, entre deux systèmes mutuellement exclusifs. Les fondateurs d'un ordre scientifique hérétique rompent le contrat d'échange qu'acceptent au moins tacitement les candidats à la succession: ne reconnaissant d'autre principe de légitimation que celui qu'ils entendent imposer, ils n'acceptent pas d'entrer dans le cycle de l'échange de reconnaissance qui assure une transmission réglée de l'autorité scientifique entre les tenants et les prétendants (c'est-à-dire, bien souvent, entre membres de générations différentes, ce qui porte beaucoup d'observateurs à réduire les conflits de légitimité à des conflits de génération). Refusant toutes les cautions et toutes les garanties qu'offre l'ordre ancien et la participation (progressive) au capital collectivement garanti qui s'opère selon les procédures réglées d'un contrat de délégation, ils réalisent l'accumulation initiale par un coup de force et par une rupture en dé-

23. Sur l'action de "filtrage» des comités de rédaction de revues scientifiques, voir D. Crane, «The Gate-Keepers of Science: Some Factors affecting the Selection of Articles for Scientific Journals », American Sociologist, II, 1967, pp. 195-201. Tout autorise à penser qu'en matière de production scientifique comme en matière de production littéraire, les auteurs sélectionnent, consciemment ou inconsciemment, les lieux de publication en fonction de l'idée qu'ils se font de leurs "normes". Tout incline à penser que l'auto-élimination, évidemment moins directement perceptible, est au moins aussi importante que l'élimination expresse (sans parler de l'effet que produit l'imposition d'une norme du publiable). 
tournant à leur profit le crédit dont bénéficiaient les anciens dominants sans leur concéder la contrepartie de reconnaissance que leur accordent ceux qui acceptent de s'insérer dans la continuité d'une lignée ${ }^{24}$.

Et tout porte à croire que la propension aux stratégies de conservation ou aux stratégies de subversion est d'autant moins indépendante des dispositions à l'égard de l'ordre établi que l'ordre scientifique est lui-même moins indépendant de l'ordre social dans lequel il se trouve inséré. C'est pourquoi on est fondé à supposer que la relation qu'établit Lewis Feuer entre les inclinations universitairement et politiquement subversives du jeune Einstein et son entreprise scientifiquement révolutionnaire vaut en quelque sorte a fortiori pour des sciences comme la biologie ou la sociologie qui sont loin d'être parvenues au degré d'autonomie de la physique du ternps d'Einstein ${ }^{25}$. Et l'opposition qu'établit cet auteur entre les dispositions révolutionnaires d'Einstein, membre dans sa jeunesse d'un groupe d'étudiants juifs en révolte contre l'ordre universitaire et l'ordre social, et les dispositions réformistes dont fait preuve Poincaré, parfait représentant de la « république des professeurs $\%$, homme d'ordre et de réforme ordonnée, tant dans l'ordre politique que dans l'ordre scientifique, ne peut manquer d'évoquer l'opposition homologue entre Marx et Durkheim.

5. Quelles sont les conditions sociales qui doivent être remplies pour que s'instaure un jeu social où l'idée vraie est dotée de force parce que ceux qui y participent ont intérêt à la vérité au lieu d'avoir, comme en d'autres jeux, la vérité de leurs intérêts? Il va de soi qu'il ne s'agit pas de faire de cet univers social

24. On verra plus loin la forme originale que revêt cette transmission réglée du capital scientifique dans les champs où, comme dans la physique aujourd'hui, la conservation et la subversion sont quasi indiscernables.

25. «Einstein's high interval of original thought was sustained by a strange little circle of young intellectuals, filled with emotions of social and scientific generational rebellion, a couriter-community of scientists outside the official scientific establishment, a group of cosmopolitan bohemians, moved in a revolutionary time to see the world in a new way (L.S. Feuer, "The Social Roots of Einstein's Theory of Relativity ", Annales of Science, vol. $27, \mathrm{n}^{\circ} 3$, September 1971, pp. 278-298 et $\mathrm{n}^{\circ} 4$, December 1971, pp. 313-344). Dépassant l'opposition naive entre les habitus individuels et les conditions sociales de leur accomplissement, Lewis Feuer suggère l'hypothèse que tous les travaux récents sur le système d'enseignement scientifique viennent corroborer (cf. M. de Saint Martin, Les fonctions sociales de l'enseignement scientifique, Paris, Lattaye-Mouton, coll. Cahiers du Centre de Sociologie européenne, $\mathrm{n}^{\circ}$ 8, 1971 et $\mathrm{P}$. Bourdieu et M. de Saint Martin, Le système des grandes écoles et la reprodicction de la classe dominante, à paraître), selon laquelle l'accès facile et rapide aux responsabilités administratives qui s'offrait en France aux élèves des grandes écoles scientifiques tendait à décourager les dispositions révolutionnaires qui trouvent au contraire un terrain favorable dans les groupes d'intellectuels marginaux, placés dans des positions de porte-à-faux entre le système d'enseignement et la bohême révolutionnaire: "One might indeed venture the hypothesis that precisely because France was a 'republic' of professors', precisely because the ablest talent of the Ecole Polytechnique was promptly absorbed into the military and engineering cadres, that it was less likely that a very foundamental break with the received principles would take place there. A scientific revolution evidently finds its most fertile soil in a counter-community. Where administrative responsibilities soon beckoned the young scientist, his energies were less available for sublimation in radical research curiosity. As far as revolutionary creativity was concerned, the very openness of the French administration to scientific talent was perhaps more important for explaining its scientific conservatism than other factors that have usually been emphasized. » 
d'exception une exception aux lois fondamentales de tout champ - et en particulier à la loi de l'intérêt qui peut conférer une violence impitoyable aux luttes scientifiques les plus «désintéressées 》 (le «désintéressement 》n'étant jamais, on l'a vu, qu'un système d'intérêts spécifiques - artistiques, religieux aussi bien que scientifiques - qui implique l'indifférence relative aux objets ordinaires de l'intérêt, argent, honneurs, etc.). Le fait que le champ scientifique comporte toujours une part d'arbitraire social dans la mesure où il sert les intérêts de ceux qui, dans le champ et/ou hors du champ, sont en mesure d'en percevoir les profits, n'exclut pas que, sous certaines conditions, la logique propre du champ et en particulier la lutte entre les dominants et les nouveaux entrants et la censure croisée qui en résulte n'exercent un détournement systématique des fins qui fait tourner continûment la poursuite des intérêts scientifiques privés (entendus toujours au double sens) au profit du progrès de la science 20 .

Les théories partielles de la science et de ses transformations sont prédisposées à remplir des fonctions idéologiques dans les luttes à l'intérieur du champ scientifique (ou de champs prétendant à la scientificité comme celui des sciences sociales) parce qu'elles universalisent les propriétés attachées à des états particuliers du champ scientifique : il en est ainsi de la théorie positiviste qui confère à la science le pouvoir de résoudre toutes les questions qu'elle pose, pourvu qu'elles soient scientifiquement posées, et d'imposer, par l'application de critères objectifs, le consensus sur ses solutions, inscrivant ainsi le progrès dans la routine de la «science normale » et faisant comme si l'on passait d'un système à un autre - de Newton à Einstein par exemple - par simple accumulation de connaissances, par affinement des mesures et par rectification des principes; il en va de même de la théorie de Kuhn, qui, valant pour les révolutions inaugurales de la science commençante (dont la révolution copernicienne fournit le paradigme - au sens vrai du mot) prend tout simplement le contrepied du modèle positiviste ${ }^{2 \tau}$. En fait, le champ de l'astronomie dans lequel survient la révolution copernicienne s'oppose au champ de la physique contemporaine à la façon dont le marché «immergé dans des relations sociales» (embedded in social relationships) des sociétés archaïques s'oppose, selon Polanyi, au «marché auto-réglé 》 (self-regulatingmarket) des sociétés capitalistes. Ce n'est pas par hasard que la révolution copernicienne implique la revendication expresse de l'autonomie pour un champ scientifique encore «immergé » dans le champ religieux et dans le champ de la philosophie et, par leur intermédiaire, dans le champ politique, revendication qui implique

26. C'est un tel mécanisme qui tend à assurer le contrôle des relations avec l'univers extérieur, avec les laïcs, c'est-à-dire la "vulgarisation scientifique * comme auto-divulgation du savant (cf. L. Boltanski et P. Maldidier, loc. cit.).

27. Il ne fait pas de doute en effet que la philosophie de l'histoire de la science que propose Kuhn, avec l'alternance de concentration monopolistique (paradigme) et de révolution, doit beaucoup au cas particulier de la "révolution copernicienne » telle qu'il l'analyse et qu'il considère comme "typique de tout autre renversement majeur de la science" (T. Kuhn, La révolution copernicienne, Paris, Fayard, 1973, p. 153 et 162) : l'autonomie relative de la science par rapport au pouvoir et en particulier par rapport à l'Eglise étant encore très faible, la révolution scientifique (dans l'astronomie mathématique) passe par la révolution politique et suppose une révolution de toutes les disciplines scientifiques qui peut avoir des effets politiques. 
l'affirmation du droit des scientifiques à trancher les questions scientifiques ( $\ll$ les mathématiques aux mathématiciens $»)$ au nom de la légitimité spécifique que leur confère leur compétence.

Tant que la méthode scientifique et la censure et/ou l'assistance qu'elle propose ou impose ne sont pas objectivées dans des mécanismes et dans des dispositions, les ruptures scientifiques prennent nécessairement l'allure de révolutions contre l'institution. Au contraire, lorsque se trouve exclu, grâce à ces révolutions criginaires, tout recours à des armes ou à des pouvoirs, même purement symboliques, autres que ceux qui ont cours dans le champ, c'est le fonctionnement même du champ qui définit de plus en plus complètement non seulement l'ordre ordinaire de la «science normale», mais aussi les ruptures extra-ordinaires, ces «révolutions ordonnées », comme dit Bachelard, qui sont inscrites dans la logique de l'histoire de la science, c'est-à-dire de la polémique scientifique ${ }^{28}$. Lorsque la méthode est inscrite dans les mécanismes du champ, la révolution contre la science instituée s'opère avec l'assistance d'une institution qui fournit les conditions institutionnelles de la rupture ; le champ devient le lieu d'une révolution permanente, mais de plus en plus totalement dénuée d'effets politiques. C'est pourquoi cet univers de la révolution permanente peut être aussi sans contradiction celui du « dogmatisme légitime ${ }^{29}$ : l'équipement scientifique qui est nécessaire pour faire la révolution scientifique ne peut être acquis que dans et par la cité scientifique. À mesure qu'augmentent les ressources scientifiques accumulées, le capital scientifique incorporé qui est nécessaire pour se les approprier et avoir ainsi accès aux problèmes et aux instruments scientifiques, donc à la lutte scientifique, devient de plus en plus important (droit d'entrée) ${ }^{30}$. Il s'ensuit que la révolution scientifique n'est pas l'affaire des plus démunis, mais au contraire des plus riches scientifiquement parmi les nouveaux entrants ${ }^{31}$. L'antinomie de la rupture et de la continuité s'affaiblit dans un champ qui, ignorant la distinction entre les phases révolutionnaires et la

28. Outre Bachelard et Reif (déjà cités), D. Bloor a aperçu que les transformations dans l'organisation sociale de la science ont déterminé une transformation de la nature des révolutions scientifiques (cf. D. Bloor, «Essay Review ; Two Paradigms for Scientific Knowledge ? ", Science Studies, 1971, 1, pp. 101-115).

29. G. Bachelard, Le Matérialisme rationnel, Paris, P.U.F., 1953, p. 41.

30. La principale censure est constituée par ce droit d'entrée, c'est-à-dire par les conditions d'accès au champ scientifique et au système d'enseignement qui $y$ donne accès. Il y aurait lieu de s'interroger sur les propriétés que les sciences de la nature (sans parler des sciences de l'homme où, du fait de la faiblesse des méthodes, toute liberté est laissée aux habitus) doivent à leur recrutement social, c'est-à-dire, grosso modo, aux conditions d'accès à l'enseignement supérieur (cf. M. de Saint Martin, op. cit.).

31. On sait que les révolutions inaugurales elles-mêmes qui donnent naissance à un nouveau champ en constituant, par la rupture, un nouveau domaine d'objectivité, incombent presque toujours à des détenteurs d'un grand capital spécifique qui, en vertu de variables secondaires (telles que l'appartenance à une classe sociale ou à une ethnie improbable dans cet univers) se trouvent placés dans une position de porte-à-faux propre à favoriser l'inclination révolutionnaire : c'est le cas par exemple des nouveaux entrants qui importent dans un champ le capital accumulé dans un champ scientifique socialement supérieur (cf. J. BenDavid, "Roles and Innovation in Medicine", American Journal of Sociology, 65, 1960, pp. 557-568; J. Ben-David et R. Collins, "Social factors in the Origins of a New Science : the Case of Psychology ", American Sociological Review, 31, 1966, pp. 451-465). 
《science normale », trouve dans la rupture continue le vrai principe de sa continuité; et corrélativement l'opposition entre les stratégies de succession et les stratégies de subversion tend de plus en plus à perdre son sens puisque l'accumulation du capital nécessaire à l'accomplissement des révolutions et du capital que procurent les révolutions tend toujours davantage à s'accomplir selon les procédures réglées d'une carrière ${ }^{32}$.

La transmutation de l'antagonisme anarchique des intérêts particuliers en dialectique scientifique devient de plus en plus totale à mesure que l'intérêt qu'a tout producteur de biens symboliques à produire des produits «qui ne soient pas seulement intéressants pour lui-même, comme dit Fred Reif, mais aussi importants pour les autres », donc propres à obtenir des autres qu'ils reconnaissent leur importance et l'importance de leur auteur, se heurte à des concurrents plus capables de mettre les mêmes moyens au service des mêmes intentions - ce qui conduit, de plus en plus fréquemment, avec les découvertes simultanées, au sacrifice de l'intérêt de l'un des producteurs ou des deux ${ }^{33}$; ou, en d'autres termes, à mesure que l'intérêt privé que chaque agent singulier a à combattre et à dominer ses concurrents pour obtenir d'eux la reconnaissance se trouve armé de tout un ensemble d'instruments qui confèrent sa pleine efficacité à son intention polémique tout en lui donnant la portée universelle d'une censure méthodique. Et, de fait, à mesure que s'accroissent les ressources accumulées et le capital nécessaire pour se les approprier, le marché sur lequel peut être placé le produit scientifique ne cesse de se restreindre à des concurrents de plus en plus fortement armés pour le critiquer rationnellement et discréditer son auteur: l'antagonisme qui est au principe de la structure et du changement de tout champ tend à devenir de plus en plus radical et de plus en plus fécond parce que l'accord forcé où s'engendre la raison laisse de moins en moins de place à l'impensé de la doxa. L'ordre collectif de la science s'élabore dans et par l'anarchie concurrentielle des actions intéressées, chaque agent se trouvant dominé - et avec lui tout le groupe - par l'entrecroisement en apparence incohérent des stratégies individuelles. C'est dire que l'opposition entre les aspects «fonctionnels 》 et les aspects «dysfonctionnels》 du fonctionnement d'un champ scientifique doté d'une grande autonomie n'a guère de sens: les tendances les plus «dysfonctionnelles » (par exemple la propension au secret et au refus de coopération) sont inscrites dans les mécanismes mêmes qui engendrent les dispositions les plus «fonctionnelles». À mesure que la méthode scientifique s'inscrit dans les mécanismes sociaux qui règlent le fonctionnement du champ et qu'elle se trouve ainsi dotée de l'objectivité supérieure d'une loi sociale immanente, elle peut réellement s'objectiver dans des instruments capables de contrôler et parfois de dominer ceux qui les utilisent et dans les dispositions durablement constituées que produit l'institution scolaire. Et ces dispositions trouvent un renfor-

32. On a vu ci-dessus la description que donne $F$. Reif de la forme que prend le plus souvent l'accumulation de capital dans un tel état du champ.

33. On s'accorde en effet pour observer que les conflits de priorité deviennent de plus en plus fréquents à mesure que la science avance, c'est-à-dire plus précisément, à mesure que les ressources scientifiques accumulées grandissent et que le capital nécessaire pour réaliser l'invention devient plus largement et uniformément répandu entre les concurrents du fait de l'élévation du droit d'entrée dans le champ. 
cement continu dans les mécanismes sociaux qui, rencontrant eux-mêmes un soutien dans le matérialisme rationnel de la science objectivée et incorporée, produisent contrôle et censure, mais aussi invention et rupture ${ }^{34}$.

6. La science n'a jamais d'autre fondement que la croyance collective dans ses fondements que produit et suppose le fonctionnement même du champ scientifique. L'orchestration objective des schèmes pratiques inculqués par l'enseignement explicite et par la familiarisation qui constitue le fondement du consensus pratique sur les enjeux proposés par le champ, c'est-à-dire sur les problèmes, les méthodes et les solutions immédiatement aperçus comme scientifiques, trouve elle-même son fondement dans l'ensemble des mécanismes institutionnels qui assurent la sélection sociale et scolaire des chercheurs (en fonction par exemple de la hiérarchie établie des disciplines), la formation des agents sélectionnés, le contrôle de l'accès aux instruments de recherche et de publication, etc. ${ }^{35}$ Le champ de discussion que dessinent, par leurs luttes, l'orthodoxie et l'hétérodoxie se découpe sur le fond du champ de la doxa, ensemble des présupposés que les antagonistes admettent comme allant de soi, en deçà de toute discussion, parce qu'ils constituent la condition tacite de la discussion ${ }^{36 ;}$ : la censure qu'exerce l'orthodoxie - et que dénonce l'hétérodoxie - cache une censure plus radicale, plus invisible aussi, parce qu'elle est constitutive du fonctionnement même du champ et qu'elle porte sur l'ensemble de ce qui est admis du seul fait de l'appartenance au champ, l'ensemble de ce qui est mis hors de discussion par le fait d'accepter les enjeux de la discussion, c'est-àdire le consensus sur les objets de dissensus, les intérêts communs qui sont au principe des conflits d'intérêts, tout l'indiscuté et l'impensé tacitement tenus hors des limites de la lutte ${ }^{3 \tau}$.

Selon le degré d'autonomie du champ par rapport aux déterminations externes, la part de l'arbitraire social qui se trouve englobée dans le système des présupposés constitutifs de la croyance propre au champ considéré est plus grande. Cela signifie que, dans l'espace abstrait de la théorie, tout champ scientifique, celui des sciences sociales ou de la mathématique aujourd'hui comme celui de

34. L'ensemble des processus qui accompagnent l'autonomisation du champ scientifique entretiennent des relations dialectiques : c'est ainsi que l'élévation continue du droit d'entrée qu'implique l'accumulation de ressources spécifiques contribue en retour à l'autonomisation du champ scientifique en instaurant une coupure sociale, d'autant plus radicale qu'elle n'est pas recherchée pour elle-même, avec le monde profane des laïs.

35. L'habitus produit par la prime éducation de classe et l'habitus secondaire inculqué par l'éducation scolaire contribuent, avec des poids différents dans le cas des sciences sociales et des sciences de la nature, à déterminer une adhésion préréflexive aux présupposés tacites du chainp (sur le rôle de la socialisation, cf. W. D. Hagstrom, op. cit. p. 9 et T. S. Kuhn, "The Function of Dogma in Scientific Research", in A. C. Crombie (ed.), Scientific Change, London, Heineman, 1963, pp. 347-369.

36. On voit ce que pourrait devenir l'ethnométhodologie (mais serait-elle encore l'ethnornéthodologie ?) si elle savait que ce qu'elle prend pour objet, le taken for granted de Schutz, c'est l'adhésion préréflexive à l'ordre établi.

37. Dans le cas du champ de production idéologique (dont participe encore le champ des sciences sociales) le fondement du consensus dans le dissensus qui définit la doxa réside, on le verra, dans la relation censurée du champ de production dans son ensemble au champ du pouvoir (c'est-à-dire dans la fonction cachée du champ de la lutte des classes). 
l'alchimie ou de l'astronomie mathématique au temps de Copernic - , peut être situé quelque part entre les deux limites représentées d'une part par le champ religieux (ou le champ de la production littéraire) dans lequel la vérité officielle n'est autre chose que l'imposition légitime (c'est-à-dire arbitraire et méconnue comme telle) d'un arbitraire culturel exprimant l'intérêt spécifique des dominants - dans le champ et hors du champ - et d'autre part par un champ scientifique d'où tout élément d'arbitraire (ou d'impensé) social serait banni et dont les mécanismes sociaux réaliseraient l'imposition nécessaire des normes universelles de la raison.

La question qui se trouve ainsi posée est celle du degré d'arbitraire social de la croyance que produit le fonctionnement du champ et qui est la condition de son fonctionnement ou, ce qui revient au même, la question du degré d'autonomic du champ (par rapport, d'abord, à la demande sociale de la classe dominante) et des conditions sociales, internes et externes, de cette autonomie. Le principe de toutes les différences entre des champs scientifiques capables de produire et de satisfaire un intérêt proprement scientifique et d'entretenir ainsi un processus dialectique interminable et des champs savants où le travail collectif n'a d'autre effet et d'autre fonction que de perpétuer le champ identique à lui-même en produisant, tant au dedans qu'au dehors, la croyance dans la valeur autonome des objectifs et des objets qu'il produit, réside dans la relation de dépendance par l'apparence d'indépendance qu'elle entretient avec les demandes externes : les doxosophes, savants apparents et savants de l'apparence, ne peuvent légitimer et la dépossession qu'ils opèrent par la constitution arbitraire d'un savoir ésotérique inaccessible au profane et la délégation qu'ils demandent en s'arrogeant le monopole de certaines pratiques ou de la réflexion sur ces pratiques, qu'à condition d'imposer la croyance que leur fausse science est parfaitement indépendante des demandes sociales qu'elle ne satisfait si bien que parce qu'elle affirme hautement son refus de les servir.

De Heidegger parlant des masses et des élites dans le langage hautement euphémisé de l'《authentique » et de l'《inauthentique » aux politicologues américains reproduisant la vision officielle du monde social dans les semi-abstractions d'un discours descriptif-normatif, c'est toujours la même stratégie de fausse coupure qui définit le jargon savant par opposition au langage scientifique. Là où le langage scientifique met des guillemets, comme l'observe Bachelard, pour marquer que les mots du langage ordinaire ou du langage scientifique antérieur qu'il conserve sont complètement redéfinis et ne tiennent leur sens que du nouveau système théorique ${ }^{38}$, le langage savant n'use des guillemets ou des néologismes que pour manifester symboliquement une distance et une rupture fictives par rapport au sens commun : ne disposant d'aucune autonomie réelle, il ne peut en effet produire complètement son effet idéologique que s'il reste assez transparent pour continuer à évoquer l'expérience et l'expression ordinaire qu'il dénie ${ }^{30}$.

Les stratégies de fausse coupure expriment la vérité objective de champs qui ne disposent que d'une fausse autonomie: en effet, tandis que la classe dominante accorde aux sciences de la nature, - aujourd'hui complètement autonomisées,

38. G. Bachelard, op. cit., pp. 216-217.

39. Cette rhétorique de la fausse coupure fera l'objet de deux analyses actuellement en préparation, l'une sur la philosophie de Heidegger, l'autre sur la politologie. 
même pour la conscience religieuse, par rapport aux lois du monde social -, une autonomie qui est à la mesure de l'intérêt qu'elle trouve dans les applications à l'économie des techniques scientifiques, elle n'a rien à attendre des sciences sociales, sinon, au mieux, une contribution particulièrement précieuse à la légitimation de l'ordre établi et un renforcement de l'arsenal des instruments symboliques de domination. Le développement tardif et toujours menacé des sciences sociales est là pour témoigner que le progrès vers l'autonomie réelle qui est la condition de l'instauration des mécanismes constitutifs d'un champ scientifique auto-réglé et autarcique se heurte nécessairement à des obstacles inconnus ailleurs : et il ne peut en être: autrement parce que l'enjeu de la lutte interne pour l'autorité scientifique dans le champ des sciences sociales, c'est-à-dire pour le pouvoir de produire, d'imposer et d'inculquer la représentation légitime du monde social, est un des enjeux de la lutte entre les classes dans le champ politique ${ }^{40}$. Il s'ensuit que les positions dans la lutte interne ne peuvent jamais atteindre le degré d'indépendance à l'égard des positions dans la lutte externe qui s'observe dans le champ des sciences de la nature. L'idée d'une science neutre est une fiction, et une fiction intéressée, qui permet de donner pour scientifique une forme neutralisée et euphémisée, donc particulièrement efficace symboliquement parce que particulièrement méconnaissable, de la représentation dominante du monde social ${ }^{41}$. En portant au jour les mécanismes sociaux qui assurent le maintien de l'ordre établi et qui doivent leur efficacité proprement symbolique à la méconnaissance de leur logique et de leurs effets, fondement d'une reconnaissance subtilement extorquée, la science sociale prend nécessairement parti dans la lutte politique. C'est dire que, lorsqu'elle parvient à. s'instaurer (ce qui suppose remplies certaines conditions, corrélatives d'un état déterminé du rapport de forces entre les classes), la lutte entre la fausse science des doxosophes (qui peuvent se réclamer des traditions théoriques les plus révolutionnaires) et la science authentique apporte nécessairement une contribution à la lutte entre les classes qui, au moins en ce cas, n'ont pas également intérêt à la vérité scientifique ${ }^{42}$.

La question fondamentale de la sociologie de la science prend, dans le cas des sciences sociales, une forme particulièrement paradoxale : quelles sont les conditions sociales de possibilité du développement d'une science affranchie des contraintes et des demandes sociales, sachant que, dans ce cas, les progrès dans le sens de la rationalité scientifique ne sont pas des progrès dans le sens de la neutralité

40. C'est ainsi que les systèmes de classement (taxinomies) sociaux, qui sont un des enjeux essentiels de la lutte idéologique entre les classes (cf. P. Bourdieu et L. Boltanski, «Le titi:e et le poste : rapports entre le système de production et le système de reproduction ", Actes de la recherche en sciences sociales, $\mathrm{n}^{\circ} 2,1975$, pp. 95-107) constituent aussi - à travers les prises de position sur l'existence ou la non-existence des classes sociales — un des grands principes de division du champ sociologique (cf. P. Bourdieu, "Classes et classements ", Minuit, 5, 1973, pp. 22-24, et A.P.A. Coxon and C.L. Jones, Occupational Categorization and Images of Society, Working Paper $\mathrm{n}^{\circ} 4$, Project on Occupational Cognition, Edinburgh, Edinburgh University Press, 1974).

41. Il s'ensuit que la sociologie de la science (et, en particulier, de la relation que la science sociale entretient avec la classe dominante), n'est pas une spécialité parmi d'autres mais qu'elle fait partie des conditions d'une sociologie scientifique.

42. Cf. P. Bourdieu, «La théorie » (entretien), in $V H 101, \mathrm{n}^{\circ} 2$, Été 1970, pp. 13-21. 
politique ? On peut nier la question. C'est ce que font par exemple tous ceux qui imputent toutes les particularités des sciences sociales à leur situation de dernières venues, au nom d'une philosophie naïvement évolutionniste qui met la science officielle au terme de l'évolution. En fait, la théorie du retard n'est vraie, paradoxalement, que dans le cas de la sociologie officielle et, plus précisément de la sociologie officielle de la sociologie. Il suffit en effet d'avoir en mémoire les analyses célèbres d'Alexander Gerschenkron sur le « retard économique », pour comprendre les traits les plus caractéristiques de ces formes particulières de discours savant que sont les fausses sciences (would-be science et science to-be). Gerschenkron remarque en effet que quand le processus d'industrialisation démarre en retard, il présente des différences systématiques avec ce qu'il a été dans les pays plus développés, non seulement en ce qui concerne la vitesse du développement, mais aussi en ce qui concerne les «structures productives et organisationnelles » cela parce qu'il met en œuvre des «instruments institutionnels » orginaux et qu'il se développe dans un climat idéologique différent ${ }^{43}$. L'existence de sciences plus avancées - grandes pourvoyeuses non seulement de méthodes et de techniques le plus souvent employées en dehors des conditions techniques et sociales d'application, mais aussi d'exemples - est ce qui permet à la sociologie officielle de se donner toutes les apparences de la scientificité : la parade de l'autonomie peut prendre ici une forme sans précédent, dont l'ésotérisme savamment entretenu des vieilles traditions lettrées ne représente qu'une pauvre anticipation. La sociologie officielle vise, non à se réaliser comme science, mais à réaliser une image officielle de la science (que la sociologie de la science contribue pour une part importante à lui fournir). La sociologie officielle de la science, sorte d'instance juridique que se donne la communauté (le mot s'applique parfaitement en ce cas) des sociologues officiels a pour fonction non seulement de fournir une idéologie justificatrice à cette communauté mais aussi et surtout de lui imposer le respect des normes et des modèles empruntés aux sciences de la nature - au prix d'une réinterprétation positiviste.

La première de ces fonctions n'est jamais aussi apparente que dans l'histoire sociale de la science sociale telle qu'elle se pratique dans l'establishment américain ${ }^{44}$. Pour convaincre complètement de la fonction d'idéologie justificatrice que remplit cette histoire sociale des sciences sociales, il suffirait de recenser l'ensemble des travaux directement ou indirectement consacrés à la compétition, le mot clé de toute la sociologie de la science américaine qui, dans son obscurité de concept indigène promu à la dignité scientifique, condense tout l'impensé (la doxa) de cette sociologie. La thèse selon laquelle productivité et compétition sont directement

43. A. Gerschenkron, Economic Backwardness in Historical Perspective, Cambridge, Harvard University Press, 1962, p. 7.

44. La philosophie de l'histoire qui hante cette histoire sociale de la science sociale trouve une expression paradigmatique dans l'ouvrage de Terry Clark que, dans un compterendu, Paul Vogt caractérise sociologiquement en deux adjectifs : "Terry N. Clark's .longawaited, much circulated in manuscript Prophets and Patrons" (cf. T. Clark, Prophets and Patrons, The French University and the Emergence of the Social Science, Cambridge (Mass.), Harvard University Press, 1973, et J.C. Chamboredon, "Sociologie de la sociologie et intérêts sociaux des sociologues", Actes de la recherche en sciences sociales, ${ }^{\circ} 2,1975$, pp. 2-17). 
liées ${ }^{45}$ s'inspire d'une théorie fonctionnaliste de la compétition qui est une variante sociologique de la croyance aux vertus du «marché libre », le mot anglais competition désignant ce que nous appelons concurrence - ; réduisant toute compétition à la compétition entre universités ou faisant de la compétition entre universités la condition de la compétition entre chercheurs, on ne s'interroge jamais sur les obstacles à la compétition scientifique qui sont imputables à la compétition à la fois économique et scientifique dont l'academic market place est le lieu. Soit cette notation d'un observateur américain de l'université anglaise : «Without intense interpersonal competition with prizes to be won, most scientists simply get on with their research and do not spend a significant part of their time thinking about where they will move next ${ }^{45 A}$ ).

La compétition que reconnaît cette science d'établissement c'est la compétition dans les formes et selon les normes de la libre entreprise intellectuelle: cette compétition dans les limites de la bienséance sociale fait d'autant plus fortement obstacle à la véritable compétition scientifique, qui met en question l'orthodoxie et, toutes les fois qu'il se peut, la doxa -, que l'on se situe dans un univers plus chargé d'arbitraire social ${ }^{46}$. On comprend que l'exaltation de l'unanimité du «paradigme » puisse coïncider avec l'exaltation de la compétition - ou encore que l'on puisse, selon les auteurs, reprocher à la sociologie européenne de pécher par excès ou par défaut de compétition.

La deuxième fonction, qui consiste à fournir les instruments et surtout les attributs symboliques de la respectabilité scientifique, masques et postiches tels que les gadgets technologiques et le kitsch rhétorique, n'est pas moins évidente. Outre les outils et les techniques - ordinateurs et programmes de traitement automatique de données par exemple -, la sociologie officielle emprunte un modèle de la pratique scientifique telle que l'imagination positiviste se la représente et un modèle de l'organisation de ce qu'elle appelle «la communauté scientifique » telle que sa pauvre science des organisations lui permet de la concevoir ${ }^{47}$. Mais la sociologie officielle n'a pas le monopole des lectures intéressées de l'histoire de la science : la difficulté particulière que la sociologie a à penser scientifiquement la science n'est pas sans rapport avec le fait qu'elle est située au plus bas de la hiérarchie sociale des sciences. Soit qu'elle s'élève pour penser des sciences plus scientifiques mieux qu'elles ne se pensent soit qu'elle s'abaisse pour enregistrer l'image triomphante que l'hagiographic scientifique produit et propage, elle a toujours la même

45. Joseph Ben-David a le mérite de donner à cette thèse sa forme la plus directe : le haut degré de compétition qui caractérise l'université américaine explique sa plus grande productivité scientifique et sa plus grande flexibilité (J. Ben-David, "Scientific productivity and academic organization in nineteenth century medicine ", American Sociological Review, $\mathrm{n}^{\circ}$ 25, 1960, pp. 828-843; Fundamental Research and the Universities, Paris, OCDE, 1968 ; J. Ben-David and Awraham Zloczower, "Universities and academic systems in modern societies ", European Journal of Sociology, n 3, 1962, pp. 45-84).

45. A. J. Gaston, "The Reward System in British Science ", American Sociological Revieu", vol. $35, n^{\circ} 4$, août 1970 .

46. Et corrélativement, cette théorie molle de la compétition qui est commune à tous les auteurs américains constitue l'obstacle le plus subtil à la construction du champ scientifique comme tel, c'est-à-dire comme lieu de lutte.

47. Sur la distorsion que la méthodologie sociologique fait subir à la vérité épistémologique de la pratique scientifique dans les sciences de la nature, voir P. Bourdieu, J.C. Chamboredon et J.C. Passeron, Le métier de sociologue, Paris, Monton-Bordas, 1968, 430 p. 
peine à se penser comme science, c'est-à-dire à penser sa position dans la hiérarchie sociale des sciences. Cela se voit en toute clarté dans les réactions qu'a suscitées le livre de Thomas Kuhn, The Structure of Scientific Revolutions et qui fourniraient un matériel expérimental de grande qualité pour une analyse empirique des idéologies de la science et de leurs relations avec la position de leurs auteurs dans le champ scientifique. Il est vrai que ce livre, dont on ne sait jamais exactement s'il décrit ou prescrit la logique du changement scientifique (exemple de prescription larvée: l'existence d'un paradigme est un signe de maturité scientifique), invitait ses lecteurs à y chercher des réponses à la question de la bonne ou de la mauvaise science ${ }^{48}$. Du côté de ceux que le langage indigène appelle des « radicaux», on a lu dans le livre de Thomas Kuhn une invitation à la « révolution » contre le «paradigme ${ }^{49}$ ou une justification du pluralisme libéral des world-views ${ }^{50}$, deux prises de position correspondant sans doute à des positions différentes dans le champ ${ }^{51}$. Du côté des tenants de l'ordre scientifique établi, on y a lu une invitation à arracher la sociologie à la phase «pré-paradigmatique » en lui imposant la constellation unifiée de croyances, de valeurs et de techniques que symbolise la triade capitoline de Parsons et de Lazarsfeld réconciliés en Merton. L'exaltation de Ja quantification, de la formalisation et de la neutralité éthique, le dédain de la «philosophie » et le rejet de l'ambition systématique au profit de la minutie de la vérification empirique et de la molle conceptualisation dite opératoire des «théories à moyenne portée $»$, autant de traits obtenus par unc transmutation désespérément transparente de l'être en devoir-être, qui trouvent leur justification dans la nécessité de contribuer au renforcement des «valeurs communautaires » tenu pour la condition du « décollage ».

Fausse science destinée à produire et à entretenir la fausse conscience, la sociologie officielle (dont la politologie est aujourd'hui le plus beau fleuron) doit faire parade d'objectivité et de «neutralité éthique » (c'est-à-dire de neutralité dans la lutte entre les classes dont elle nie par ailleurs l'existence) et donner toutes les apparences d'une coupure tranchée avec la classe dominante et ses demandes idéo-

48. Plus encore que dans ce livre - dont les thèses essentielles n'ont rien de radicalement nouveau, au moins pour les lecteurs de Bachelard, objet lui-même, à peu près au même moment, d'une semblable captation, dans une autre tradition l'intention normative se voit dans deux articles où $T$. Kuhn décrit les fonctions positives pour le développement scientifique d'une pensée "convergente" et soutient que l'adhésion dogmatique à une tradition est favorable à la recherche (T. Kuhn, "The Function of Dogma in Scientific Research" in A.C. Crombie (ed.), op. cit., pp. 347-369; "The Essentail Tension : Tradition and Innovation in Scientific Research , in L. Hudson (ed.), The Ecology of Human Intelligence, London, Penguin, 1970, pp. 342-359).

49. Cf. par exemple, R.W. Friedrichs, A Sociology of Sociology, New York, Free Press, 1970.

50. E. Gellner, "Myth, Ideology and Revolution ", in B. Crick, and W.A. Robson (ed.), Protest and Discontent, London, Penguin, 1970, pp. 204-220.

51. Une revue telle que Theory and Society doit limportance purement sociale qui lui permet d'exister et de subsister sans autre contenu positif que cette sorte de vague humanisme antipositiviste auquel se reconnaissent les "sociologues critiques " (autre concept indigène), au fait qu'elle donne une unité strictement négative à tous les courants qui se trouvent ou se pensent hors de l'establishment américain, depuis l'ethnométhodologie, héritière de la phénoménologie, au néo-marxisme, en passant par la psychohistory. (On trouvera un tableau synoptique assez fidèle de cette constellation idéologique in $\mathbf{P}$. Bandyapadhyav, "One Sociology or Many : Some Issues in Radical Sociology ", Sociological Review, vol. 19, February 1971, pp. 5-30). 
logiques en multipliant les signes extérieurs de scientificité : on a ainsi, du côté "empirique », la parade technologique et du côté de la « théorie », la rhétorique $d u$ «néo» (florissante aussi dans le champ artistique) qui signe la cumulativité scientifique en appliquant à une œuvre ou à un ensemble d'œuvres du passé (cf. The Structure of Social Action) la procédure typiquement lettrée de la « relecture », opération paradigmatiquement scolaire de simple reproduction ou de reproduction simple bien faite pour produire, dans les limites du champ et de la croyance qu'il produit, toutes les apparences de la « révolution». Il faudrait analyser systématiquement cette rhétorique de la scientificité par laquelle la «communauté » dominante produit la croyance dans la valeur scientifique de ses produits et dans l'autorité scientifique de ses membres : soit par exemple l'ensemble des stratégies destinées à donner les apparences de la cumulativité, telles que la référence aux sources canoniques, le plus souvent réduites, comme on dit, "à leur plus simple expression» (que l'on pense au destin posthume du Suicide) c'est-à-dire à de plats protocoles simulant la froide rigueur du discours scientifique, et aux articles autant que possible récents (on connaît l'opposition entre les sciences dures - hard - et les sciences «molles»- soft--), sur le même sujet; ou encore les stratégies de fermeture, qui entendent marquer une séparation tranchée entre la problématique scientifique et les débats profanes et mondains (toujours présents, mais au titre de 《fantômes dans la machine »), cela au prix le plus souvent, de simples retraductions linguistiques; ou les stratégies de dénégation, qui fleurissent chez les politologues, habiles à réaliser l'idéal dominant de «l'objectivité » dans un discours apolitique sur la politique où la politique refoulée ne peut apparaître que sous les apparences méconnaissables, donc irréprochables, de sa dénégation politologique ${ }^{52}$. Mais ces stratégies remplissent par surcroît une fonction essentielle : la circulation circulaire des objets, des idées, des méthodes et surtout des signes de reconnaissance à l'intérieur d'une communauté (il faudrait dire un club ouvert aux seuls membres indigènes ou importés de l'Ivy League) ${ }^{53}$ produit, comme tout cercle de légitinité, un univers de croyance dont on trouve l'équivalent aussi bien dans le champ religieux que dans le champ de la littérature ou de la haute couture ${ }^{54}$.

Mais, ici encore, il faut se garder de conférer à la fausse science officielle la signification que lui confère la critique « radicale ». Malgré leur opposition sur la valeur qu'ils confèrent au «paradigme», principe d'unification nécessaire au développement de la science dans un cas, force de répression arbitraire dans l'autre cas, - ou tour à tour l'un ou l'autre chez Kuhn -, conservateurs et « radicaux », adversaires complices, s'accordent en fait sur l'essentiel : par le point de vue unilatéral qu'ils prennent nécessairement sur le champ scientifique en choisissant au moins inconsciemment l'un ou l'autre des camps antagonistes, ils ne peuvent aper-

52. Cf. P. Bourdieu, "Les doxosophes , Minuit, 1, 1973, pp. 26 45 (en particulier l'analyse de l'effet Lipset).

53. La sociologie officielle de la science offre une justification pour chacun de ces traits. Ainsi par exemple, l'évitement des problèmes théoriques fondamentaux trouve une justification dans l'idée que dans les sciences de la nature, les chercheurs ne s'inquiètent pas de la philosophie de la science (cf. W.O. Hagstrom, op. cit., pp. 277-279). On voit sans peine ce qu'une telle sociologie de la science peut devoir à la nécessité de légitimer un état de fait et de transformer les limites subies en exclusions électives.

54. Sur la production de la croyance et du fétichisme dans le champ de la haute couture, voir P. Bourdieu et Y. Delsaut, « Le couturier et sa griffe : contribution à une théorie de la magie ", Actes de la recherche en sciences sociales, $\mathrm{n}^{\circ} 1$, janvier 1975, pp. 7-36. 
cevoir que le contrôle ou la censure n'est pas exercée par telle ou telle des instances mais par la relation objective entre adversaires complices qui, par leur antagonisme même, délimitent le champ de la discussion légitime, excluant comme saugrenu ou éclectique, ou tout simplement impensable, toute tentative pour prendre une position non prévue (dans le cas particulier, pour mettre par exemple au service d'une.autre axiomatique scientifique les outils techniques élaborés par la science officielle) ${ }^{55}$.

Expression à peine euphémisée des intérêts des dominés du champ scientifique, l'idéologie «radicale» tend à traiter toute révolution contre l'ordre scientifique établi comme révolution scientifique, faisant comme s'il suffisait qu'une «innovation » soit rejetée par la science officielle pour qu'elle puisse être tenue pour scientifiquement révolutionnaire et omettant ainsi de poser la question des conditions sociales par lesquelles une révolution contre l'ordre scientifique établi est inséparablement une révolution scientifique et non une simple hérésie visant à renverser le rapport des forces établi dans le champ sans transformer les principes sur lesquels repose son fonctionnement ${ }^{5 t}$. Quant aux dominants, inclinés à admettre que l'ordre scientifique dans lequel sont placés tous leurs investissements (au sens de l'économie et de la psychanalyse) et dont ils sont en mesure de s'approprier les profits, est le devoir-être réalisé, ils sont logiquement portés à adhérer à la philosophie spontanée de la science qui trouve son expression dans la tradition positiviste, forme de l'optimisme libéral qui veut que la science progresse par la force intrinsèque de l'idée vraie et que les «puissants » soiẹnt aussi par définition les plus «compétents ${ }^{57}$ : il suffit de penser à des états anciens du champ des sciences de la nature ou à l'état actuel du champ des sciences sociales pour apercevoir la fonction idéologique de sociodicée de cette philosophie de la science qui, en donnant l'idéal pour réalisé, exclut la question des conditions sociales de réalisation de l'idéal.

En posant que la sociologie de la science elle-même fonctionne selon les lois de fonctionnement de tout champ scientifique qu'établit la sociologie scientifique de la science, la sociologie de la science ne se condamne nullement au relativisme. En effet, une sociologie scientifique de la science (et la sociologie scientifique qu'elle contribue à rendre possible) ne peut se constituer qu'à condition d'apercevoir

55. De tels couples épistémologiques, qui sont en même temps des couples sociologiques, fonctionnent en tout champ (cf. par exemple le Positivismusstreit qui oppose Habermas et Popper dans le cas de l'Allemagne - mécanisme de détournement qui ayant fait ses preuves en Europe commence à sévir aux USA avec l'importation de l'école de Francfort).

56. Il faudrait analyser tous les usages stratégiques que les dominés dans un champ peuvent faire de la transfiguration idéologique de leur position objective : par exemple, la parade de l'exclusion qui permet aux exclus de tirer encore parti de l'institution (qu'ils reconnaissent assez pour lui reprocher de ne pas les avoir reconnus) en faisant de l'exclusion une garantie de scientificité ; ou encore la contestation de la "compétence 》 des dominants qui est au centre de tout mouvement hérétique (cf. la contestation du monopole du sacrement) et qui doit d'autant moins s'armer d'arguments scientifiques que le capital scientifique accumulé est plus faible, etc.

57. Proposition qui est vraie sociologiquement, mais qui se réduit alors à une simple tautologie, la "compétence " ne désignant rien d'autre que l'autorité socialement reconnue, celle du théologien de Sorbonne au Moyen-Age aussi bien que celle du prix Nobel de physique aujourd'hui. 
clairement qu'aux différentes positions dans le champ scientifique sont associées des représentations de la science qui sont autant de stratégies idéologiques et de prises de position épistémologiques par lesquelles les occupants d'une position déterminée visent à justifier leur propre position et les stratégies qu'ils mettent en œuvre pour la maintenir ou l'améliorer en même temps qu'à discréditer les tenants de la position opposée et leurs stratégies. Chaque sociologue est bon sociologue de ses concurrents, la sociologie de la connaissance ou de la science n'étant que la forme la plus irréprochable des stratégies de disqualification de l'adversaire aussi longtemps qu'elle prend pour objet les adversaires et leurs stratégies et non le système complet des stratégies, c'est-à-dire le champ des positions à partir duquel elles s'engendrent ${ }^{58}$. La sociologie de la science n'est si difficile que parce que le sociologue a des enjeux dans le jeu qu'il prétend décrire (soit premièrement la scientificité de la sociologie et deuxièmement la scientificité de la forme de la sociologie qu'il pratique) et qu'il ne peut objectiver ces enjeux et les stratégies correspondantes qu'à condition de prendre pour objet non les seules stratégies de ses adversaires scientifiques mais le jeu en tant que tel, qui commande aussi ses propress stratégies, menaçant de gouverner souterrainement sa sociologie.

\title{
RéSUMIÉ
}

En opposition à la sociologie officielle de la science qui ne fait qu'enregistrer l'image triomphante que l'hagiographie scientifique produit et propage, qui constitue la « communauté scientifique " comme un champ social d'exception et qui néglige les phénomènes de domination, l'auteur introduit et élabore la notion de " champ scientifique » qu'il définit à la fois comme le système des relations objectives entre des positions sociales et comme le lieu de la concurrence qui a pour enjeu spécifique le monopole de l'autorité scientifique inséparablement définie comme capacité technique et comme pouvoir social. Il découle de cette définition qu'il est vain de distinguer entre des déterminations proprement scientifiques et des déterminations proprement sociales des pratiques scientifiques : la lutte pour l'autorité scientifique est une lutte irréparablement politique et scientifique dont la spécificité est qu'elle oppose des producteurs qui tendent à n’avoir d'autres clients que leurs concurrents.

\begin{abstract}
Opposing the official sociology of science which does nothing more than legitimate the triumphant image that the scientific priesthood produces and propagates, which sees the "scientific community" as an exceptional social environment and which neglects the phenomenon of domination, the author introduces and elaborates the notion of "scientific field" which he defines both as a system of objective relations between social positions and as a place of competition for the specific prize of a monopoly as scientific authority defined both as technical ability and social power. This definition means that one cannot distinguish between causes of scientific practices that are strictly scientific and those that are strictly social: The struggle for scientific authority is necessarily a struggle which is both political and scientific. Its only uniqueness is that it pits producers against each other who tend to have no other clients than their competitors.
\end{abstract}

58. Sur la nécessité de construire comme tel le champ intellectuel pour rendre possible une sociologie des intellectuels qui soit autre chose qu'un échange d'injures et d'anathèmes entre "intellectuels de droite" et "intellectuels de gauche ", voir P. Bourdieu. "Les fractions de la classe dominante et les modes d'appropriation de l'oeuvre d'art ", Information sur les sciences sociales, 13 (3), 1974, pp. 7-32. 


\section{RESUMEN}

En oposición a la sociología oficial que no hace más que registrar la imagén triunfante que la hagiografia científica produce y propaga, que constituye la "communidad cientifíca " como un campo social de excepción y que no presta mucha atención a los fenómenos de dominación, el autor introduce y elabora la noción de "campo científico" y la define a la vez como el lugar de la cometencia que tiene como encuentro (enjeu) específico el monopolio de la autoridad científica inseparablemente definida como capacidad técnica y como poder social. Lo que se desprende de esta definición es que es vano distinguir entre las determinaciones propiamente científicas y las determinaciones propiamente sociales de las prácticas científicas : la lucha, por la autoridad científica es una lucha irreparablemente política y científica, su caracter especifico es que opone los productores que tienden a no tener otros clientes sino sus competidores. 